\title{
Cancerona: Challenges of Cancer Management in Times of COVID-19 Pandemic
}

\author{
Farah Ballout $^{1} \cdot$ Reem Daouk ${ }^{1} \cdot$ Joseph Azar ${ }^{1} \cdot$ Michael Timonian $^{1} \cdot$ Tarek Araji $^{1} \cdot$ Hisham F. Bahmad $^{1,2}$ (D) \\ Wassim Abou-Kheir ${ }^{1}$
}

Accepted: 24 September 2020 / Published online: 30 September 2020

(C) Springer Nature Switzerland AG 2020

\begin{abstract}
Coronavirus disease 2019 (COVID-19) has infected millions of people worldwide and emerged to be the biggest global health threat claiming hundreds of thousands of lives at exponential rates. The severity of the disease increases with old age and presence of underlying health conditions, such as cancer. Managing cancer patients under these circumstances is rather challenging, given their compromised immunity and the overwhelmed health care services by COVID-19 community transmission. Thus, it is prudent to establish common guidelines for the monitoring and treatment of cancer patients. In this review, we comprehensively investigate the various aspects of cancer care during the COVID-19 pandemic, discuss challenges faced while treating cancer patients, and propose potential approaches to manage COVID-19 among this vulnerable population. We also discuss molecular aberrations and genetic changes associated with cancer and their role in affecting the virus' infectivity and severity. Lastly, we shed light on therapeutic approaches that can encompass both diseases without compromising one over the other.
\end{abstract}

Keywords COVID-19 $\cdot$ SARS-CoV-2 $\cdot$ Cancer $\cdot$ Immune response $\cdot$ Therapeutic target

\section{Introduction}

Reports of a new acute respiratory illness caused by a new strain of coronavirus, the severe acute respiratory syndrome coronavirus 2 (SARS-CoV-2), have appeared in late December 2019 in Wuhan, China [1, 2]. The disease was announced to be a global pandemic in March 2020 and was termed COVID-19 by the World Health Organization (WHO) [3]. SARS-CoV-2 has infected more than 33 million

This article is part of the Topical Collection on COVID-19

Hisham F. Bahmad

hfbahmad@gmail.com; Hisham.Bahmad@msmc.com

Wassim Abou-Kheir

wa12@aub.edu.lb

1 Department of Anatomy, Cell Biology, and Physiological Sciences, Faculty of Medicine, American University of Beirut, Beirut, Lebanon

2 Arkadi M. Rywlin M.D. Department of Pathology and Laboratory Medicine, Mount Sinai Medical Center, 4300 Alton Rd, Miami Beach, FL 33140, USA individuals and caused nearly 1 million deaths worldwide by the end of September 2020 [3]. COVID-19 manifests commonly with mild respiratory symptoms including cough and shortness of breath, accompanied by a moderate to high fever. However, the severity of this disease can widely range from asymptomatic to pneumonitis requiring hospitalization and oxygen support to respiratory dysfunction and multipleorgan failure and ultimately death $[1,2]$.

Coronaviruses are enveloped single-stranded RNA viruses that cause respiratory, enteric, hepatic, and neurological diseases [4]. Of these species, four are prevalent and associated with common cold symptoms in immunocompetent individuals [5] and two strains originate from animals and have been linked to severe acute respiratory syndrome outbreaks including severe acute respiratory syndrome coronavirus (SARS$\mathrm{CoV}$ ) in 2002 in Guangdong Province, China and Middle East respiratory syndrome coronavirus (MERS-CoV) in 2012 in the Middle East [6, 7].

The unprecedented occurrence of the current coronavirus pandemic is having a profound effect on entire communities and not the least the oncology community. Cancer affects a significant portion of the population globally and cancer patients are more susceptible to infections due to their systemic 
immunosuppressive state caused by the malignant tumor itself and anticancer treatments [8,9]. As a consequence, cancer patients are predisposed to SARS-CoV-2 infection and are more likely to have higher mortality than the general population [10]. A study showed that cancer patients had double the risk of SARS-CoV-2 infection compared with the general population [11]. Overall, providing care to immunocompromised cancer patients amidst this pandemic has proven to be extremely challenging not just because of increased risk of infection and mortality, but because of an increased need for mechanical ventilation or ICU admission concomitant with limited resources in outpatient settings, including administrative staff and specialists $[12,13]$. In this review, we aimed to look at various aspects and consequences of the COVID-19 pandemic on cancer patients from a global standpoint, and to discuss potential treatment options for this vulnerable subgroup.

\section{Response of Cancer Care Units to the Global Pandemic of COVID-19}

\section{Preparedness and Management of Different Cancer Types During the COVID-19 Pandemic}

After the massive mobilization of health care resources to control the COVID-19 pandemic and mitigate its repercussions, it has become evident that many concerns were raised regarding the provision and preparedness of cancer care units. One of the most worrisome aspects has been the impact that COVID-19 may have on cancer therapeutic strategies and clinical pathways, knowing that the uncertainty, unexpectedness, and prompt expansion are three crucial characteristics of the pandemic. Although the European Society of Medical Oncology's (ESMO) recent guidelines highlighted the primordial need to prioritize the treatment of cancer patients that constitute a vulnerable population [14], the global health care sector's preparedness was a major limitation. In one of the largest medical centers in Spain, the oncology department witnessed a heavy decline in its activity. As compared with the same period in 2019, there was a decline of $23 \%$ in the number of outpatient's visits, $37 \%$ in new oncology referrals, and $43 \%$ in the number of patients enrolled in clinical trials. These numbers underline a serious lack of preparedness that would worsen the delay in diagnosis and treatment of cancer patients and would ultimately result in deleterious survival [15]. It is true that the unexpected personal protective equipment (PPE) shortage crisis also reflected the deficiency in preparing for and facing the pandemic. Some governmental agencies in the US recommended using homemade PPE, but this alone would neglect the fact that cancer patients undergoing chemotherapy are probably one of the most vulnerable subpopulations of COVID-19 patients [16]. In conclusion, the optimal strategy to compensate for the lack of preparedness should focus on raising efforts to establish an adequate adaptation. The important transitional adaptations that were employed in cancer care will be highlighted in the next sections of this review.

As previously noted, a drastic adjustment in the management of different cancer types was needed to overcome the impact of the growing pandemic. Notable changes were applied in the management of both solid and hematological malignancies. When it comes to breast cancer, for instance, ESMO classified treatment approaches as high-, medium-, and low-priority based on the burden of the disease and the clinical stability of the patient. The American College of Surgeons (ACS) assessed health care institutions based on the availability of resources and the load of COVID-19 patients. When it comes to screening, the American Society of Breast Surgeons (ASBrS) and the American College of Radiology (ACR) issued a joint statement in which they recommended postponing all imaging studies until the COVID19 period ends, as the exposure cost outweighs the benefit of screening during this short period [17]. It is also important to acknowledge that there is a rising call to standardize the management procedures amidst the danger of potentially exposing vulnerable cancer patients to the virus. This explains the subsequent increase in the usage of online telehealth modalities [18].

For gastrointestinal malignancies, although there is moderate evidence establishing that delayed resection of colorectal cancer leads to a worse prognosis, it is uncertain whether delaying pancreatic and gastric surgeries has the same outcome. During the COVID-19 pandemic, a caseby-case methodology should be employed to account for the benefit of doing early resections that achieve curative outcomes [19]. When it comes to head and neck neoplasms, in under 2 weeks, 30 international radiation oncology experts were involved in a Delphi consensus process that leads to the publication of a new international guideline that deals with the radiotherapy of patients with head and neck cancers during the COVID-19 period. The Head and Neck International Group is currently developing an international consensus guideline to further enhance the decision-making ability of health care providers [20]. Notable efforts were also demonstrated by a panel of international experts that issued multiple recommendations concerning lung cancer radiotherapy. Briefly, their recommendations mainly revolved around the following: intensive screening, making use of hypofractionation, delaying post-operative radiation therapy, and limiting exposure by avoiding twice-daily treatments. They also recommended delaying prophylactic cranial irradiation or delivering it simultaneously with radiotherapy or radiochemotherapy for limited-stage small cell lung cancer. 


\section{COVID-19 Pandemic: a Tough Hit on the Vulnerable}

The major shift in cancer management was indeed a crucial step that was globally taken. However, one must admit that the pandemic had an enormous impact on cancer patients despite the multidisciplinary efforts that were assembled. With a 30 day all-cause mortality that has been shown to be higher in patients with neoplasms and COVID-19, the vulnerability of this population of patients stands as a threatening element. In fact, not only is this mortality associated with general risk factors but it is also associated with risk factors that are only present in patients who have cancer [21]. The fact that patients with cancer and COVID-19 have worse clinical outcomes than patients with COVID-19 alone has been heavily discussed [22]. One has to remember that special care and intensive coordination should be employed in middle- to low-income countries where financial constraints further accentuate the burden of cancer patients [23]. To note, many published recommendations for the management of cancer patients during the pandemic took into account the financial burdens by carefully assessing the applicability in low- and middle-income regions [24]. One example that demonstrates the vulnerability of cancer patients is the detrimental repercussions of having false negative nasopharyngeal swabs. One may cautiously conclude that during the pandemic, cancer patients with flu-like symptoms and pathognomonic radiological findings should be treated as if they have COVID-19, irrespective of their swab results [25]. Despite the tough hit that cancer patients are enduring during the pandemic, the battle is still ongoing. In one tertiary care in Italy - which is considered one of the main virus hotspots in Europe and the world - major cancer surgeries and transplantations were still being prioritized and performed despite the heavy burden of the pandemic [26]. Perhaps new approaches in cancer care must be developed to keep the battle going: media campaigns, evidence-based screening programs, prioritizing and anticipating cancer surgeries, and benchmarking cancer outcomes are all potential key approaches that may be applied [27].

\section{A Battle with the Unknown: a Call for Innovation}

Undoubtedly, the pandemic exerted a heavy pressure on the global health care sector by unexpectedly depleting its human and medical resources. As paradoxical as it may sound, this pressure may have benefited the medical field in a way. As a matter of fact, dealing with an unknown opponent necessitated using innovative approaches and modalities. Despite the global stellar efforts that were highlighted in this review, uncertainty stood as a challenging opponent for humanity during the pandemic. Until vaccination becomes the norm and more efficient therapeutic strategies are employed, cancer patients should be supplied with resources that help them in coping with their heavy burden and in enhancing their cooperation with their health care providers [28].

\section{Clinical Effects of COVID-19 on Cancer Patients}

With the emergence of the SARS-CoV-2, it has become clear that certain populations are particularly vulnerable to severe infections and poor clinical outcomes, and cancer patients are on the top of the list. This is due to the immunosuppressive state caused by the malignancy and chemotherapy drugs [13]. In addition, cancer patients above 60 years old are particularly susceptible to severe infections and high mortality rates compared with their younger counterparts [11]. Even though respiratory symptoms are the most common signs of infection, the virus is capable of multiorgan damage since its receptorangiotensin-converting enzyme 2-is located in several organs [29].

\section{COVID-19 Testing in Cancer Patients}

Reverse transcriptase polymerase chain reaction (RT-PCR) is the standard diagnostic test used to detect SARS-CoV-2, and a nasopharyngeal swab is the most common method used to obtain an adequate sample [30]. Its main drawback, however, is its high false negative rates, which undermines the true number of affected individuals [31]. This, in turn, has highlighted the need to repeat testing. In fact, a study on RTPCR testing for COVID-19 showed that the test might turn positive after two consecutive negative results in up to $20 \%$ of patients [32]. Serologic testing has been proposed as another method to detect SARS-CoV-2. A recent analysis demonstrated that seroconversion occurred in $50 \%$ of patients 7 days after infection, and in $100 \%$ of patients 14 days after infection [33]. These antibodies are detected even if the RT-PCR results were negative. Nonetheless, it has not been encouraged to rely solely on serological testing to diagnose SARS-CoV-2 because even though antibodies are detected in the serum, their generation, long-term immunity, and possible cross-reactivity with other coronavirus strains are not yet properly understood [34].

Even though SARS-CoV-2 infection can cause an array of symptoms, asymptomatic infections are not uncommon [35]. Standard testing in some areas are reserved for symptomatic patients and people with possible exposure to COVID-19, thus undermining the true number of SARS-CoV-2 carriers. Since asymptomatic patients are capable of transmitting the virus, this testing approach might fail to properly protect vulnerable populations such as cancer patients [34]. To minimize infection rates in cancer patients, leading centers have encouraged testing for SARS-CoV-2 before initiating antineoplastic treatments including systemic treatments, radiation therapies, and surgeries [34]. 


\section{Clinical Characteristics and Chest CT Findings of Cancer Patients with COVID-19}

Zhang et al. conducted a multicenter retrospective study to determine the clinical outcomes of cancer patients infected with COVID-19 [36]. The study included 107 cancer patients with various neoplasms infected with the novel coronavirus. Almost half developed a severe infection after contracting COVID-19. The common presenting signs of infection were fever, cough, fatigue, and dyspnea, which are similar symptoms seen in non-immunocompromised patients. Thirty-seven out of the total 107 patients were receiving treatments at the time of their diagnosis. They were found to have a higher risk of severe infection and mortality rate compared with those not receiving current treatment. They were also more likely to have abnormal laboratory tests such as lymphopenia and elevated inflammatory markers $(\mathrm{C}$-reactive protein and procalcitonin), and they required mechanical ventilation more often. A variety of other complications were reported, including acute respiratory stress syndrome and heart failure [36]. In addition, patients who received cancer treatment within 14 days of COVID-19 diagnosis were more likely to develop severe events such as admission to the intensive care unit, use of assisted mechanical ventilation or even death [37].

Even though anticancer treatments predispose cancer patients to severe SARS-CoV-2 infections, delaying treatment is not always a valid option. Nagar et al. argued that patients receiving radiotherapy should continue with their treatment regimen despite the risk of SARS-CoV-2 infection [38]. Delaying adjuvant radiotherapy in breast cancer patients after surgery was found to be associated with $50 \%$ chance of breast cancer recurrence [39]. Similarly, head and neck cancer patients were found to have a lower overall survival rate when the interval between surgery and radiotherapy was prolonged [40]. Moreover, radiotherapy does not exploit hospital resources such as hospital beds and ventilators and traditional radiation schedules can be modified by replacing them with shorter and hypofractionated sessions or administering fewer sessions leading to less immunosuppression with similar clinical outcomes [38].

Imaging is of particular importance in both diagnosis and monitoring the progression of COVID-19 infection. Typical findings include bilateral peripheral ground-glass opacities (GGOs) with possible consolidation and vascular ectasia [41]. Imaging, however, might not always be helpful in cancer patients infected with COVID-19. For example, lung cancer patients infected with SARS-CoV-2 may show GGOs and consolidations, which cannot always be differentiated from the primary lung tumor or other opportunistic infections [42]. Imaging in patients with acute myeloid leukemia and lymphomas infected with SARS-CoV-2 can also be inconclusive. CT images may show interstitial and alveolar infiltrations in addition to GGOs, findings also manifested in other conditions that frequently occur in leukemic patients such as acute respiratory stress syndrome and alveolar hemorrhage [43]. Therefore, additional diagnostic tests such as RT-PCR are needed in case COVID-19 infection is suspected in cancer patients [42].

\section{Cancer Screening in the COVID-19 Era}

Cancer screening has been majorly affected during the pandemic. The governmental restrictions on commuting as well as the need to control the spread of the virus have halted screening procedures [44]. This approach, however, may have long-term consequences.

Colorectal cancer (CRC) screening, for instance, usually starts at the age of 50 for both men and women. Patients first undergo a fecal occult blood test (FOBT); if the test is positive, then they are scheduled for a colonoscopy. The steps that follow are dictated by the findings of the colonoscopy. Screening for colorectal cancer has reduced the overall mortality of CRC by detecting neoplasms at an early stage [45]. In the case of a positive FOBT, the earlier the follow up with a colonoscopy the better the clinical outcomes are. In fact, multiple studies have demonstrated that delaying a colonoscopy after a positive FOBT can have unfavorable effects on the overall survival and mortality risk of patients [46]. Since FOBT is not being utilized during the epidemic, the cases of undiagnosed CRCs will not only increase in the absence of screening, but will also be detected at late stages [47]. This in turn might cause a surge in the mortality rate of CRC patients [48] and put a financial strain on health care systems, as advanced CRCs have higher costs than early-stage neoplasms [49]. One way to overcome these limitations according to Blanco et al. is to prioritize colonoscopy to high-risk patients such as those with inflammatory bowel disease (IBD), a strong family history of $\mathrm{CRC}$, or documented positive FOBT. Screening in asymptomatic patients or patients with no immediate alarming symptoms on the other hand may be delayed. The date of the last colonoscopy and relevant findings are also important to note. This strategy could be effective in ensuring that high-risk patients receive proper treatment as early as possible [44].

Lung cancer screening was also affected by the COVID-19 pandemic. Low-dose chest $\mathrm{CT}$ is used for screening high-risk patients, especially those with a smoking history. In case a nodule is detected, its size and characteristics are noted. A panel of twenty-four experts including pulmonologists, thoracic surgeons and thoracic radiologists proposed guidelines to manage lung cancer screening during the pandemic. The consensus is to delay non-urgent cases till after the pandemic, while providing adequate care to urgent ones [50]. Interestingly, the panel recommended that treatment for stage I non-small cell lung cancer can be delayed after careful assessment of its characteristics including its size, growth rate, 
PET scan results, and patient's preference. These recommendations are general guidelines and should be considered in the context of several factors including but not limited to SARS$\mathrm{CoV}-2$ prevalence in the community, commuting guidelines imposed by the government, the availability of resources, and the overall burden of the COVID-19 pandemic.

\section{Effect of COVID-19 on Cancer Clinical Trials}

The SARS-CoV-2 pandemic has also had a detrimental effect on cancer clinical trials, which are currently around 12,000 trials [51]. Resources including equipment and research staff were reassigned to assist hospitals and institutions in managing the large influx of infected patients. Cancer clinical trials and patient recruitment have been stopped, and research efforts and funding were redirected to study SARS-CoV-2. Conferences and staff training were also halted and postponed. Moreover, data collection and entry from ongoing clinical trials were delayed [52]. The financial impact of the pandemic on clinical trials is also significant. It is estimated that on average a phase III oncology clinical trial can cost up to 20 million USD [53]. Restarting a clinical trial after restrictions are lifted can add to the overall cost and put additional strain on previous clinical trials.

These restrictions will have long-term consequences on cancer research. There will be a delay in the delivery of potential drugs and therapies with promising clinical outcomes. One example is the ASCOLT trial, which recruited over 1480 patients in 12 Asian countries to evaluate the role of Aspirin in high-risk colorectal cancer patients. The rapid spread of SARS-CoV-2 in these regions has caused patient recruitment and trials to stop, and scheduled visits were canceled [54].

\section{Role of Molecular Aberrations and Genetic Changes Associated with Cancer in COVID-19}

It has been reported that cancer predisposes individuals to SARS-CoV-2 infection and poor prognosis due to the malignant tumor itself, chemotherapy, and surgery. Coronavirus infectivity depends on the binding of its viral spike (S) protein to angiotensin-converting enzyme 2 (ACE2) receptor and its priming by the type II transmembrane serine protease (TMPRSS2) to allow fusion of viral and host cellular membranes $[29,55]$. ACE2 is a blood pressure regulator through the renin-angiotensin-aldosterone system and is expressed in the lungs, kidneys, prostate, and intestines, suggesting susceptibility of these organs to SARS-CoV-2 infection. The viral spike (S) protein of SARS-CoV-2 consists of two subunits: S1 and $\mathrm{S} 2$. The $\mathrm{S} 1$ subunit mediates viral infectivity by binding to ACE2, and the S2 subunit mediates the membrane fusion process [56]. Investigating the interaction of the S2 subunit with other proteins that have role in cancer would provide an insight into the mechanism and severity of COVID-19 in cancer patients. Singh et al. [57] showed that the S2 subunit of SARS-CoV-2 strongly interacts with the well-known tumor suppressor proteins, $\mathrm{p} 53$ and BRCA-1/2. These proteins regulate various downstream signaling pathways and are frequently mutated in human cancer suggesting the need for further research to understand COVID-19 effect in cancer patients [58].

Analyzing the expression of ACE2 in various tumor tissues has been also used to evaluate the susceptibility of these patients to SARS-CoV-2 infection. Ahmadi et al. [59] showed that ACE2 mRNA and protein levels were higher in colon adenocarcinoma (COAD) patients than in the control group, suggesting more susceptibility to COVID-19. This upregulation was not correlated with patient survival outcomes. Further assessment showed that the ACE2 gene promoter is hypomethylated in COAD patients and the methylation status is negatively correlated with ACE2 expression. On the other hand, $A C E 2$ gene expression positively correlated with infiltration level of immune cells including memory B cell, plasma $\mathrm{B}$ cell, activated myeloid dendritic cell, and resting myeloid dendritic cell in COAD patients [59]. SARS-CoV-2 infection was shown to reduce ACE2 level post-infection [58], which would bring poor prognosis for patients with colon cancer due to altered immune cell infiltration level. In a landscape profiling analysis on the expression level of ACE2 in pan-cancers, Dai et al. [60] showed that $A C E 2$ was significantly differentially expressed in six types of cancer. It was elevated in breast invasive carcinoma (BRCA) and liver hepatocellular carcinoma (LIHC) and downregulated in kidney chromophobe $(\mathrm{KICH})$, prostate adenocarcinoma (PRAD), thyroid carcinoma (THCA), and stomach adenocarcinoma (STAD) [60]. In addition, Zhang et al. [61] demonstrated significantly elevated $A C E 2$ expression in 10 cancer types, including kidney renal papillary cell carcinoma (KIRP), kidney renal clear cell carcinoma (KIRC), COAD, pancreatic adenocarcinoma (PAAD), STAD, esophageal carcinoma (ESCA), lung adenocarcinoma (LUAD), lung squamous carcinoma (LUSC), cervical squamous cell carcinoma and endocervical adenocarcinoma (CESC), and adrenocortical carcinoma (ACC) [61]. Similarly, Chai et al. [62] performed a systematic analysis of ACE2 RNA expression and DNA methylation across over 30 tumors. ACE2 was upregulated in COAD, KIRP, PAAD, rectum adenocarcinoma (READ), STAD, and LUAD. This upregulation was associated with epigenetic modification of $A C E 2$ in these tumors, namely hypomethylation [62]. Another study by Cai et al. [63] reported increased expression of ACE2 in ESCA, KIRP, LUAD, and uterine corpus endometrial carcinoma (UCEC) and high TMPRSS2 levels in $\mathrm{KICH}, \mathrm{PRAD}$, and uterine corpus endometrial carcinoma (UCEC); hence, higher risk of COVID-19 infection especially in UCEC patients co-expressing both ACE2 and TMPRSS2 receptors [63]. Collectively, decreased $A C E 2$ expression was 
significantly associated with poor prognosis, whereas high expression indicated favorable prognosis but higher susceptibility to SARS-CoV-2. ACE2 was shown to inhibit breast cancer angiogenesis through suppressing VEGFa/VEGFR2/ ERK pathway [64] and to reduce cell invasion and migration and inhibit EMT in non-small cell lung cancer (NSCLC) cells [65], which could explain its regulatory effect in tumorigenesis.

Following the same approach, Katopodis et al. [66] analyzed the expression and DNA methylation of TMPRSS2 and cathepsin L (CTSL), a host cell protease that facilitates $\mathrm{S}$ protein cleavage. TMPRSS2 was overexpressed in CESC, COAD, PRAD, READ, UCEC, and uterine carcinosarcoma. CTSL was upregulated in lymphoid neoplasm diffuse large B cell lymphoma, esophageal carcinoma, glioblastoma multiforme, head and neck squamous cell carcinoma, lower grade glioma, pancreatic adenocarcinoma, skin cutaneous melanoma, stomach adenocarcinoma, and thymoma. This upregulation was associated with hypomethylation of both genes [66].

TMPRSS2 gene expression is promoted through androgen receptor and increases upon exposure to androgens [55]. The androgen-regulated gene TMPRSS 2 has been reported in the pathophysiology of about $50 \%$ of prostate cancers when fused with the oncogenic ETS transcription factor $E R G$ where it has been shown to attenuate androgen signaling through direct inhibition of $A R$ expression and downregulating $A R$ signaling at gene-specific loci [67]. Androgen deprivation therapy (ADT) has been shown to significantly downregulate TMPRSS2 in patients with TMPRSS2:ERG fusion expressing prostate cancer thus possibly reducing infectivity and severity of SARS-CoV-2 in these patients. These results might indicate modulation of TMPRSS2 expression in human lungs by ADT as a therapeutic strategy against COVID-19 [68, 69]. A study by Montopoli et al. [70] supported this finding by showing that prostate cancer patients receiving ADT had a significantly lower risk of SARS-CoV-2 infection compared with patients who did not receive ADT. In contrast, Koskinen et al. [71] results did not support a role of ADT in decreasing the risk of SARS-CoV-2 infection in men with prostate cancer via ADTmediated decrease in the expression of TMPRSS2. This discrepancy was explained by methodological differences mainly the region of data acquisition. Further investigation of the impact of ADT on TMPRSS2 and ACE2 is certainly needed prior to clinical application.

Epithelial to mesenchymal transition (EMT) is a wellcharacterized phenomenon co-opted by tumor cells to promote resistance and metastasis [72]. Stewart et al. [58] showed that $A C E 2$ expression is restricted to a population of highly epithelial cells and infection of lung cancer models with SARS-CoV-2 upregulates established EMT regulators, miR200 family - zinc finger E-box-binding homeobox 1 (ZEB1) and TAM family receptor tyrosine kinase (AXL) - thereby downregulating ACE2 post-infection. Their work suggested a novel model for SARS-CoV-2 pathogenesis in which the virus initially infects highly epithelial $A C E 2$ expressing cells in the aerodigestive and respiratory tracts followed by EMTlike molecular changes shifting the infected cells toward an increasingly mesenchymal state, which in turn results in the loss of $A C E 2$ expression, along with its acute respiratory distress syndrome-protective effect [58].

A major characteristic of patients with severe COVID-19 infection is the inflammatory immune response that potentiates a cytokine storm involving the upregulation of tumor necrosis factor- $\alpha(\mathrm{TNF} \alpha)$, interleukin $1 \beta$ (IL- $1 \beta)$, IL-6, monocyte chemoattractant protein-1 (MCP-1), and macrophage inflammatory proteins $1-\alpha(\operatorname{MIP} 1 \alpha)$. These inflammatory reactions contribute to multiorgan dysfunction, tissue damage and may promote tumor progression [68]. The IL17 cytokine is generated in the lung in reaction to viral infection. Viral persistence leads to augmentation of IL-17 and provokes acute distress respiratory syndrome (ARDS), just as it occurs in SARS-CoV-2 infection. The role of IL-17 is well known in lung cancer, where it has been associated with increased angiogenesis and poor prognoses. Blocking IL-17 could be theoretically advantageous in subjects with SARSCoV-2 infection [73].

It is likely that cancer patients have a different susceptibility to SARS-CoV-2 infection, a different course, and a different prognosis. This highlights the value of protective actions for cancer patients during the COVID-19 pandemic era. Close attention should be paid to treating cancer patients with COVID-19 infection due to the inherent immune resistance in the tumor microenvironment, which makes it difficult to clear the SARS-CoV-2 virus from infected tumor cells.

\section{Anticancer Therapeutic Approaches in the Treatment of COVID-19}

The similarities encountered in the interactions of the human body with cancer cells and virally infected cells have led to many propositions regarding treatment of viral infections with standard cancer therapies such as chemotherapy, radiotherapy, and immunotherapy. In this section of our review, we will shed light on the potential of anticancer therapies on COVID-19 management.

\section{Immunotherapy: Interaction Between Cancer and Viral Therapy/ Radiotherapy for Viral Infection}

Immune checkpoint inhibitors (ICPIs) have been revolutionary therapeutic options for many types of cancer, such as nonsmall cell lung cancer, melanoma, Hodgkin's lymphoma, and renal cell carcinoma. ICPIs are antibodies that interrupt inhibitory cell surface receptor interactions between the tumor cells 
and cytotoxic T cells, restoring intrinsic antitumor effects and leading to more effective immune mediated cytotoxicity. ICPIs fall under the category of immunotherapy, which involves antibodies that target PD-1 on $\mathrm{T}$ cells (Pembrolizumab, Nivolumab), PD-L1/2 on tumor cells (Atezolizumab), and CTLA-4 on T cells (Ipilimumab).

In the light of the COVID-19 pandemic, many studies are being conducted to investigate the effect of ICPI therapy in cancer patients with increased risk for SARS-CoV-2 infection. In a recent review by Gambichler et al., it has been hypothesized that the use of ICPIs in viral infections should not play a harmful role given the observed similarities in immune exhaustion between malignancies and viral infections [74]. Using flow cytometry, it was shown that patients with severe manifestations of SARS-CoV-2 infection had much higher expression of PD-1 on T cells compared with healthy patients [74], indicating that $\mathrm{T}$ cell exhaustion plays a significant role in COVID-19, similar to other viral infections. Based on this observation, Chiappelli et al., entertained the possibility of using ICPIs to mitigate $\mathrm{T}$ cell exhaustion and thus reestablish $\mathrm{T}$ cell mediated cytotoxicity; however, this may only work in the early and middle stages of SARS-CoV-2 infections, because as the infection progresses PD-1 expression increases to a point where $\mathrm{T}$ cell exhaustion becomes irreversible and refractory to ICPIs [75]. Nivolumab, a PD-1 inhibitor, is being tested in a phase II randomized trial for safety and efficacy when compared with standard of care therapy for COVID-19 patients hospitalized in the ICU (NCT04343144). Moreover, viral clearance is being studied alongside safety and efficacy in adults with SARS-CoV-2 infection receiving nivolumab in an open-label, controlled, single-center pilot study (NCT04356508). According to a study by Luo et al. (2020), patients who are already receiving PD-1 inhibition ICPIs for malignancies and become infected with SARS-CoV-2 can safely continue their regimen with no increase in severity of the COVID-19 disease [76].

CIP poses several concerns when it comes to patients at risk of SARS-CoV-2 infection. First, the difficulty in differentiating CIP from newly acquired SARS-CoV-2 infection. CIP and COVID-19 infection may have very similar radiographical and clinical presentations; however, the medical management differs significantly. Second, treatment of CIP requires a combination of immunosuppressants including corticosteroids and immune modulators (anti-TNF- $\alpha$, anti-IL6 ), which may carry an increased risk of acquiring new viral infections or reactivation/dissemination of latent viral infections. To elaborate, patients with severe COVID-19 infections have been found to be in a hyperinflammatory state, described as the "cytokine storm," which has been associated with poorer outcomes and higher mortality rates, according to a review article by Calabresse et al. [77]. Anti-IL-6 treatment (Tocilizumab) was shown to be successful in many patients with severe COVID-19 infection in China, and many centers in the United States have adopted off-label use of anti-IL-6 regimens in such patients [77]. A phase II clinical trial is underway to compare anti-IL-6 treatment with standard medical management of serious COVID-19 cases with threatening respiratory failure (NCT04322773).

The effectiveness of immunotherapy in treating both cancer and viral infections does not end here. In a phase Ib open label clinical trial, researchers studied an IL-15 super agonist in combination with nivolumab for the treatment of metastatic non-small cell lung cancer. The trial demonstrated that this dual immunotherapy is safe to be administered on an outpatient basis, and most importantly can re-invoke responses in patients that have failed therapy with anti-PD1 immunotherapy alone [78]. Additionally, IL-15 immunotherapy has been proposed to be a viable treatment options for COVID-19 infections because of its role in activating the innate and adaptive immune system to clear the viral infection. IL-15 promotes the proliferation of $\mathrm{NK}$ cells, $\mathrm{CD}^{+} \mathrm{T}$ cells, and $\mathrm{T}_{\text {regulatory }}$ cells, which in turn induce rapid viral clearance. These immune cells also release IL-10 and INF- $\gamma$ that help counteract the $\mathrm{Th}_{2}$-mediated cytokine storm observed in COVID-19 infections [79].

\section{Radiotherapy for Viral Infection}

On another note, pneumonia has been historically treated with low-dose radiation therapy due to its antiinflammatory effects. This is different from the moderate to high-dose radiation that is used for cancer therapy. According to Mortazavi (2020), given the current lack of effective antiviral therapy against SARS-CoV-2 infection, low-dose radiation therapy is an effective management option for patients with moderate to severe signs of infection. Merits of low-dose radiation therapy include its antiinflammatory properties, combating cytokine storm by inducing apoptosis of cytokine-producing inflammatory cells, and preventing thromboembolic complications by decreasing blood clotting. Low-dose radiotherapy may also prevent the emergence of drug-resistant viral strains that are encountered when ineffective antiviral drugs are used. A phase II clinical trial is being conducted to assess the antiinflammatory properties of low-dose radiation for patients with SARS-CoV-2 infection with respiratory compromise. More studies are needed to delineate the benefits and safety profile of low-dose radiation therapy in treatment of inflammatory processes such as in COVID-19 disease [80].

In conclusion, an interface exists between anticancer therapy and antiviral therapy specifically for COVID-19 disease. This interface encompasses three major fronts: first, immunotherapy using immune checkpoint inhibitors, type III interferon, and Galectin-3 inhibitors; second, cytokine blockade using anti-IL-6 and anti-TNF- $\alpha$; and third, radiation therapy in differential doses. Based on the several risk factors mentioned, particular care should be given to cancer patients as 
they are at increased risk for SARS-CoV-2 infection and are often receiving chemotherapeutic regimens that put them at increased chances of adverse drug interactions should antiviral drugs be used.

\section{Conclusions and Future Directions}

The COVID-19 pandemic is potentially the greatest public health crisis in modern history. It has brought unprecedented challenges to health care systems worldwide by causing immense stress and pressure on the health care workforce. Consequently, the outbreak is yielding great challenges on cancer management that may have direct and remote effects on patients and caregivers alike. During such a crisis, listing ongoing issues may allow the oncology community to identify solutions for proper management and treatment. The higher vulnerability of patients with cancer to COVID-19 infection and severe disease prompt the generation of a comprehensive set of pragmatic approaches to manage these patients and an in-depth review of potential treatment options that take into account both diseases.

Acknowledgments We want to thank all members of the Abou-Kheir's Laboratory for their support. This work was not funded.

Code Availability Not applicable.

Author Contributions WAK and HFB conceived the concept and idea of the present review. WAK, HFB, and FB worked on the study design strategy and selected the topics to be discussed. FB, RD, JA, MT, and TA did literature searches, screened titles and abstracts for relevance, abstracted the data from the eligible full text articles, analyzed and interpreted the data, and drafted the manuscript. WAK and HFB critically revised the manuscript. All authors have read and approved the final draft.

Data Availability Not applicable.

\section{Compliance with Ethical Standards}

Conflict of Interest The authors declare that they have no conflict of interest.

Ethics Approval Not applicable.

Consent to Participate Not applicable.

Consent for Publication Not applicable.

\section{References}

1. Huang C, Wang Y, Li X, Ren L, Zhao J, Hu Y, et al. Clinical features of patients infected with 2019 novel coronavirus in Wuhan, China. Lancet. 2020;395(10223):497-506. https://doi. org/10.1016/S0140-6736(20)30183-5.
2. Zhu N, Zhang D, Wang W, Li X, Yang B, Song J, et al. A Novel Coronavirus from patients with pneumonia in China, 2019. N Engl J Med. 2020;382(8):727-33. https://doi.org/10.1056/ NEJMoa2001017.

3. World Health Organization (2020) Rolling updates on coronavirus disease (COVID-19). World Health Organization. https://www. who.int/emergencies/diseases/novel-coronavirus-2019/events-asthey-happen. Accessed 29 Sept.

4. Weiss SR, Leibowitz JL. Coronavirus pathogenesis. Adv Virus Res. 2011;81:85-164. https://doi.org/10.1016/B978-0-12-3858856.00009-2.

5. Su S, Wong G, Shi W, Liu J, Lai ACK, Zhou J, et al. Epidemiology, genetic recombination, and pathogenesis of coronaviruses. Trends Microbiol. 2016;24(6):490-502. https://doi.org/10.1016/j.tim. 2016.03.003.

6. Cui J, Li F, Shi ZL. Origin and evolution of pathogenic coronaviruses. Nat Rev Microbiol. 2019;17(3):181-92. https:// doi.org/10.1038/s41579-018-0118-9.

7. Zaki AM, van Boheemen S, Bestebroer TM, Osterhaus AD, Fouchier RA. Isolation of a novel coronavirus from a man with pneumonia in Saudi Arabia. N Engl J Med. 2012;367(19):1814 20. https://doi.org/10.1056/NEJMoa1211721.

8. Groll AH, Castagnola E, Cesaro S, Dalle JH, Engelhard D, Hope W, et al. Fourth European Conference on Infections in Leukaemia (ECIL-4): guidelines for diagnosis, prevention, and treatment of invasive fungal diseases in paediatric patients with cancer or allogeneic haemopoietic stem-cell transplantation. Lancet Oncol. 2014;15(8):e327-40. https://doi.org/10.1016/S1470-2045(14) 70017-8.

9. Torre LA, Siegel RL, Ward EM, Jemal A. Global cancer incidence and mortality rates and trends - an update. Cancer Epidemiol Biomark Prev. 2016;25(1):16-27. https://doi.org/10.1158/10559965.EPI-15-0578.

10. Dai M, Liu D, Liu M, Zhou F, Li G, Chen Z, et al. Patients with cancer appear more vulnerable to SARS-CoV-2: a multicenter study during the COVID-19 outbreak. Cancer Discov. 2020;10(6):783-91. https://doi.org/10.1158/2159-8290.CD-200422.

11. Yu J, Ouyang W, Chua MLK, Xie C. SARS-CoV-2 transmission in patients with cancer at a tertiary care hospital in Wuhan, China. JAMA Oncol. 2020;6:1108-10. https://doi.org/10.1001/ jamaoncol.2020.0980.

12. Kattan C, Badreddine H, Rassy E, Kourie HR, Kattan J. The impact of the coronavirus pandemic on the management of cancer patients in Lebanon: a single institutional experience. Future Oncol. 2020;16(17):1157-60. https://doi.org/10.2217/fon-2020-0313.

13. Liang W, Guan W, Chen R, Wang W, Li J, Xu K, et al. Cancer patients in SARS-CoV-2 infection: a nationwide analysis in China. Lancet Oncol. 2020;21(3):335-7. https://doi.org/10.1016/S14702045(20)30096-6.

14. Cancer patient management during the COVID-19 pandemic. https:/www.esmo.org/guidelines/cancer-patient-managementduring-the-covid-19-pandemic.

15. Manso L, De Velasco G, Paz-Ares L. Impact of the COVID-19 outbreak on cancer patient flow and management: experience from a large university hospital in Spain. ESMO Open. 2020;4(Suppl 2): e000828. https://doi.org/10.1136/esmoopen-2020-000828.

16. Nelson R. Lack of protective gear disrupts oncology care. Lancet Oncol. 2020;21(5):631-2. https://doi.org/10.1016/S1470-2045(20) 30223-0.

17. ASBrS and ACR Joint Statement on Breast Screening Exams During the COVID-19 Pandemic https://www.breastsurgeons.org/ docs/news/2020-03-26-ASBrS-ACR-Joint-Statement.pdf.

18. Sundriyal D, Sehrawat A, Kumar P, Bhandari R. Impact of COVID-19 pandemic on oncology practices during nationwide 
lockdown period: a single centre experience and the way forward. J Assoc Physicians India. 2020;68(7):48-50.

19. Fligor SC, Wang S, Allar BG, Tsikis ST, Ore AS, Whitlock AE, et al. Gastrointestinal malignancies and the COVID-19 pandemic: evidence-based triage to surgery. J Gastrointest Surg. 2020:1-17. https://doi.org/10.1007/s11605-020-04712-5.

20. Mehanna H, Gillison M, Lee AWM, von Zeidler SV, Porceddu S. Adapting head and neck cancer management in the time of COVID19. Int J Radiat Oncol Biol Phys. 2020;107(4):628-30. https://doi. org/10.1016/j.ijrobp.2020.04.017.

21. Kuderer NM, Choueiri TK, Shah DP, Shyr Y, Rubinstein SM, Rivera DR, et al. Clinical impact of COVID-19 on patients with cancer (CCC19): a cohort study. Lancet. 2020;395(10241):190718. https://doi.org/10.1016/s0140-6736(20)31187-9.

22. Tang LV, Hu Y. Poor clinical outcomes for patients with cancer during the COVID-19 pandemic. Lancet Oncol. 2020;21(7):862-4. https://doi.org/10.1016/S1470-2045(20)30311-9.

23. Trehan A, Jain R, Bansal D. Oncology care in a lower middleincome country during the COVID-19 pandemic. Pediatr Blood Cancer. 2020;67(8):e28438. https://doi.org/10.1002/pbc.28438.

24. Belkacemi Y, Grellier N, Ghith S, Debbi K, Coraggio G, Bounedjar A, et al. A review of the international early recommendations for departments organization and cancer management priorities during the global COVID-19 pandemic: applicability in low- and middleincome countries. Eur J Cancer (Oxford, England : 1990). 2020;135:130-46. https://doi.org/10.1016/j.ejca.2020.05.015.

25. Veccia A, Kinspergher S, Dipasquale M, Caffo O. Nasopharyngeal swab or clinical-radiological evidence: the dark side of the moon for cancer patients in the COVID-19 era. Future Oncol. 2020;16(19): 1321-2. https://doi.org/10.2217/fon-2020-0372.

26. Berardi G, Colasanti M, Levi Sandri GB, Del Basso C, Ferretti S, Laurenzi A, et al. Continuing our work: transplant surgery and surgical oncology in a tertiary referral COVID-19 center. Updat Surg. 2020;72(2):281-9. https://doi.org/10.1007/s13304-02000825-3.

27. Butler J, Finley C, Norell CH, Harrison S, Bryant H, Achiam MP, et al. New approaches to cancer care in a COVID-19 world. Lancet Oncol. 2020;21(7):e339-40. https://doi.org/10.1016/s14702045(20)30340-5.

28. Young AM, Ashbury FD, Schapira L, Scotté F, Ripamonti CI, Olver IN. Uncertainty upon uncertainty: supportive Care for Cancer and COVID-19. Support Care Cancer. 2020;28:1-4. https://doi.org/10.1007/s00520-020-05604-9.

29. Zhou P, Yang X-L, Wang X-G, Hu B, Zhang L, Zhang W, et al. A pneumonia outbreak associated with a new coronavirus of probable bat origin. Nature. 2020;579(7798):270-3. https://doi.org/10.1038/ s41586-020-2012-7.

30. Loeffelholz MJ, Tang YW. Laboratory diagnosis of emerging human coronavirus infections - the state of the art. Emerg Microbes Infect. 2020;9(1):747-56. https://doi.org/10.1080/22221751.2020. 1745095.

31. Li Y, Yao L, Li J, Chen L, Song Y, Cai Z, et al. Stability issues of RT-PCR testing of SARS-CoV-2 for hospitalized patients clinically diagnosed with COVID-19. J Med Virol. 2020;92(7):903-8. https://doi.org/10.1002/jmv.25786.

32. Xiao AT, Tong YX, Zhang S. False negative of RT-PCR and prolonged nucleic acid conversion in COVID-19: rather than recurrence. J Med Virol. 2020. https://doi.org/10.1002/jmv.25855.

33. Wolfel R, Corman VM, Guggemos W, Seilmaier M, Zange S, Muller MA, et al. Virological assessment of hospitalized patients with COVID-2019. Nature. 2020;581(7809):465-9. https://doi.org/ 10.1038/s41586-020-2196-x.

34. Madariaga A, McMullen M, Sheikh S, Kumar R, Liu FF, Zimmermann C, et al. COVID-19 testing in cancer patients: does one size fit all? Clin Cancer Res. 2020;26:4737-42. https://doi.org/ 10.1158/1078-0432.CCR-20-2224.
35. Ooi EE, Low JG. Asymptomatic SARS-CoV-2 infection. Lancet Infect Dis. 2020;20:996-8. https://doi.org/10.1016/S14733099(20)30460-6.

36. Zhang H, Wang L, Chen Y, Wu Q, Chen G, Shen X, et al. Outcomes of novel coronavirus disease 2019 (COVID-19) infection in 107 patients with cancer from Wuhan, China. Cancer. 2020;126:4023-31. https://doi.org/10.1002/cncr.33042.

37. Zhang L, Zhu F, Xie L, Wang C, Wang J, Chen R, et al. Clinical characteristics of COVID-19-infected cancer patients: a retrospective case study in three hospitals within Wuhan, China. Ann Oncol. 2020;31(7):894-901. https://doi.org/10.1016/j.annonc. 2020.03.296.

38. Nagar H, Formenti SC. Cancer and COVID-19 - potentially deleterious effects of delaying radiotherapy. Nat Rev Clin Oncol. 2020;17(6):332-4. https://doi.org/10.1038/s41571-020-0375-1.

39. Huang J, Barbera L, Brouwers M, Browman G, Mackillop WJ. Does delay in starting treatment affect the outcomes of radiotherapy? A systematic review. J Clin Oncol. 2003;21(3):555-63. https:// doi.org/10.1200/JCO.2003.04.171.

40. Harris JP, Chen MM, Orosco RK, Sirjani D, Divi V, Hara W. Association of survival with shorter time to radiation therapy after surgery for US patients with head and neck cancer. JAMA Otolaryngol Head Neck Surg. 2018;144(4):349-59. https://doi. org/10.1001/jamaoto.2017.3406.

41. Kooraki S, Hosseiny M, Myers L, Gholamrezanezhad A. Coronavirus (COVID-19) outbreak: what the Department of Radiology should know. J Am Coll Radiol. 2020;17(4):447-51. https://doi.org/10.1016/j.jacr.2020.02.008.

42. Katal S, Aghaghazvini L, Gholamrezanezhad A. Chest-CT findings of COVID-19 in patients with pre-existing malignancies; a pictorial review. Clin Imaging. 2020;67:121-9. https://doi.org/10.1016/j. clinimag.2020.06.004.

43. Potenza L, Luppi M, Morselli M, Tonelli S, D’Apollo N, Facchini $\mathrm{L}$, et al. Leukaemic pulmonary infiltrates in adult acute myeloid leukaemia: a high-resolution computerized tomography study. $\mathrm{Br}$ J Haematol. 2003;120(6):1058-61. https://doi.org/10.1046/j.13652141.2003.04192.x.

44. Del Vecchio Blanco G, Calabrese E, Biancone L, Monteleone G, Paoluzi OA. The impact of COVID-19 pandemic in the colorectal cancer prevention. Int J Color Dis. 2020;35:1951-4. https://doi.org/ 10.1007/s00384-020-03635-6.

45. Gini A, Jansen EEL, Zielonke N, Meester RGS, Senore C, Anttila A, et al. Impact of colorectal cancer screening on cancer-specific mortality in Europe: a systematic review. Eur J Cancer (Oxford, England : 1990). 2020;127:224-35. https://doi.org/10.1016/j.ejca. 2019.12.014.

46. Kim NH, Lim JW, Kim S, Lim JY, Kim W, Park JH, et al. Association of time to colonoscopy after a positive fecal test result and fecal hemoglobin concentration with risk of advanced colorectal neoplasia. Dig Liver Dis. 2019;51(4):589-94. https://doi.org/10. 1016/j.dld.2018.12.008.

47. Vicentini M, Zorzi M, Bovo E, Mancuso P, Zappa M, Manneschi $\mathrm{G}$, et al. Impact of screening programme using the faecal immunochemical test on stage of colorectal cancer: results from the IMPATTO study. Int J Cancer. 2019;145(1):110-21. https://doi. org/10.1002/ijc.32089.

48. Ladabaum U, Dominitz JA, Kahi C, Schoen RE. Strategies for colorectal cancer screening. Gastroenterology. 2020;158(2):418 32. https://doi.org/10.1053/j.gastro.2019.06.043.

49. Clerc L, Jooste V, Lejeune C, Schmitt B, Arveux P, Quantin C, et al. Cost of care of colorectal cancers according to health care patterns and stage at diagnosis in France. Eur J Health Econ. 2008;9(4):361-7. https://doi.org/10.1007/s10198-007-0083-0.

50. Mazzone PJ, Gould MK, Arenberg DA, Chen AC, Choi HK, Detterbeck FC, et al. Management of lung nodules and lung cancer screening during the COVID-19 pandemic: CHEST expert panel 
report. Chest. 2020;158(1):406-15. https://doi.org/10.1016/j.chest. 2020.04.020.

51. Richards M, Anderson M, Carter P, Ebert BL, Mossialos E. The impact of the COVID-19 pandemic on cancer care. Nat Can. 2020;1(6):565-7. https://doi.org/10.1038/s43018-020-0074-y.

52. Saini KS, de Las Heras B, de Castro J, Venkitaraman R, Poelman M, Srinivasan G, et al. Effect of the COVID-19 pandemic on cancer treatment and research. Lancet Haematol. 2020;7(6):e432-5. https://doi.org/10.1016/s2352-3026(20)30123-x.

53. Sertkaya A, Wong HH, Jessup A, Beleche T. Key cost drivers of pharmaceutical clinical trials in the United States. Clin Trials. 2016;13(2):117-26. https://doi.org/10.1177/1740774515625964.

54. Segelov E, Prenen H, Day D, Macintyre CR, Foo EMJ, Ali R, et al. Impact of the COVID-19 epidemic on a pan-Asian academic oncology clinical trial. JCO Glob Oncol. 2020;6:585-8. https://doi. org/10.1200/go.20.00072.

55. Hoffmann M, Kleine-Weber H, Schroeder S, Krüger N, Herrler T, Erichsen S, et al. SARS-CoV-2 cell entry depends on ACE2 and TMPRSS 2 and is blocked by a clinically proven protease inhibitor. Cell. 2020;181(2):271-280.e278. https://doi.org/10.1016/j.cell. 2020.02.052.

56. Xia S, Liu M, Wang C, Xu W, Lan Q, Feng S, et al. Inhibition of SARS-CoV-2 (previously 2019-nCoV) infection by a highly potent pan-coronavirus fusion inhibitor targeting its spike protein that harbors a high capacity to mediate membrane fusion. Cell Res. 2020;30(4):343-55. https://doi.org/10.1038/s41422-020-0305-x.

57. Singh N, Bharara Singh A. S2 subunit of SARS-nCoV-2 interacts with tumor suppressor protein p53 and BRCA: an in Silico study. Transl Oncol. 2020;13(10):100814. https://doi.org/10.1016/j. tranon.2020.100814.

58. Stewart CA, Gay CM, Ramkumar K, Cargill KR, Cardnell RJ, Nilsson MB, et al. SARS-CoV-2 infection induces EMT-like molecular changes, including ZEB1-mediated repression of the viral receptor ACE2, in lung cancer models. bioRxiv : the preprint server for biology. 2020. https://doi.org/10.1101/2020.05.28.122291.

59. Ahmadi M, Saffarzadeh N, Habibi MA, Hajiesmaeili F, Rezaei N. Colon cancer and SARS-CoV-2: impact of ACE2 expression in susceptibility to COVID-19. bioRxiv the preprint server for biology: 2020.2006.2011.146878. 2020. https://doi.org/10.1101/2020. 06.11 .146878 .

60. Dai YJ, Hu F, Li H, Huang HY, Wang DW, Liang Y. A profiling analysis on the receptor ACE2 expression reveals the potential risk of different type of cancers vulnerable to SARS-CoV-2 infection. Ann Transl Med. 2020;8(7):481. https://doi.org/10.21037/atm. 2020.03.61.

61. Zhang L, Han X, Shi Y. Comparative analysis of SARS-CoV-2 receptor ACE2 expression in multiple solid tumors and matched non-diseased tissues. Infect Genet Evol. 2020;85:104428. https:// doi.org/10.1016/j.meegid.2020.104428.

62. Chai P, Yu J, Ge S, Jia R, Fan X. Genetic alteration, RNA expression, and DNA methylation profiling of coronavirus disease 2019 (COVID-19) receptor ACE2 in malignancies: a pan-cancer analysis. J Hematol Oncol. 2020;13(1):43. https://doi.org/10.1186/ s13045-020-00883-5.

63. Cai C, Ahmed OA, Shen H, Zeng S. Which cancer type has the highest risk of COVID-19 infection? J Inf Secur: S01634453(0120)30307-30308. 2020. https://doi.org/10.1016/j.jinf. 2020.05.028.

64. Zhang Q, Lu S, Li T, Yu L, Zhang Y, Zeng H, et al. ACE2 inhibits breast cancer angiogenesis via suppressing the VEGFa/VEGFR2/ ERK pathway. J Exp Clin Cancer Res. 2019;38(1):173. https://doi. org/10.1186/s13046-019-1156-5.

65. $\mathrm{Xu} \mathrm{J,} \mathrm{Fan} \mathrm{J,} \mathrm{Wu} \mathrm{F,} \mathrm{Huang} \mathrm{Q,} \mathrm{Guo} \mathrm{M,} \mathrm{Lv} \mathrm{Z,} \mathrm{et} \mathrm{al.} \mathrm{The}$ ACE2/angiotensin-(1-7)/mas receptor axis: pleiotropic roles in cancer. Front Physiol. 2017;8(276). https://doi.org/10.3389/fphys. 2017.00276.

66. Katopodis P, Anikin V, Randeva HS, Spandidos DA, Chatha K, Kyrou I, et al. Pan-cancer analysis of transmembrane protease serine 2 and cathepsin $L$ that mediate cellular SARS-CoV-2 infection leading to COVID-19. Int J Oncol. 2020;57(2):533-9. https://doi. org/10.3892/ijo.2020.5071.

67. Yu J, Yu J, Mani R-S, Cao Q, Brenner CJ, Cao X, et al. An integrated network of androgen receptor, polycomb, and TMPRSS2ERG gene fusions in prostate cancer progression. Cancer Cell. 2010;17(5):443-54. https://doi.org/10.1016/j.ccr.2010.03.018.

68. Bhowmick NA, Oft J, Dorff T, Pal S, Agarwal N, Figlin RA, et al. COVID-19 and androgen targeted therapy for prostate cancer patients. Endocr Relat Cancer. 2020;27:R281-92. https://doi.org/10. 1530/erc-20-0165.

69. Bahmad HF, Abou-Kheir W. Crosstalk between COVID-19 and prostate cancer. Prostate Cancer Prostatic Dis. 2020. https://doi. org/10.1038/s41391-020-0262-y.

70. Montopoli M, Zumerle S, Vettor R, Rugge M, Zorzi M, Catapano $\mathrm{CV}$, et al. Androgen-deprivation therapies for prostate cancer and risk of infection by SARS-CoV-2: a population-based study $(\mathrm{N}=$ 4532). Ann Oncol. 2020;31:1040-5. https://doi.org/10.1016/j. annonc.2020.04.479.

71. Koskinen M, Carpen O, Honkanen V, Seppänen MRJ, Miettinen PJ, Tuominen JA, et al. Androgen deprivation and SARS-CoV-2 in men with prostate cancer. Ann Oncol. 2020;31:1417-8. https://doi. org/10.1016/j.annonc.2020.06.015.

72. Cheaito KA, Bahmad HF, Hadadeh O, Saleh E, Dagher C, Hammoud MS, et al. EMT markers in locally-advanced prostate cancer: predicting recurrence? Front Oncol. 2019;9:131. https://doi. org/10.3389/fonc.2019.00131.

73. Allegra A, Pioggia G, Tonacci A, Musolino C, Gangemi S. Cancer and SARS-CoV-2 infection: diagnostic and therapeutic challenges. Cancers. 2020;12(6). https://doi.org/10.3390/cancers12061581.

74. Gambichler T, Reuther J, Scheel CH, Becker JC. On the use of immune checkpoint inhibitors in patients with viral infections including COVID-19. J Immunother Cancer. 2020;8(2):e001145. https://doi.org/10.1136/jitc-2020-001145.

75. Chiappelli F, Khakshooy A, Greenberg G. CoViD-19 immunopathology and immunotherapy. Bioinformation. 2020;16(3):219-22. https://doi.org/10.6026/97320630016219.

76. Luo J, Rizvi H, Egger JV, Preeshagul IR, Wolchok JD, Hellmann MD. Impact of PD-1 blockade on severity of COVID-19 in patients with lung cancers. Cancer Discov. 2020;10:1121-8. https://doi.org/ 10.1158/2159-8290.Cd-20-0596.

77. Calabrese C, Rajendram P, Sacha G, Calabrese L. Practical aspects of targeting IL-6 in COVID-19 disease. Cleve Clin J Med. 2020. https://doi.org/10.3949/ccjm.87a.ccc018.

78. Wrangle JM, Velcheti V, Patel MR, Garrett-Mayer E, Hill EG, Ravenel JG, et al. ALT-803, an IL-15 superagonist, in combination with nivolumab in patients with metastatic non-small cell lung cancer: a non-randomised, open-label, phase $1 \mathrm{~b}$ trial. Lancet Oncol. 2018;19(5):694-704. https://doi.org/10.1016/s1470-2045(18) 30148-7.

79. Kandikattu HK, Venkateshaiah SU, Kumar S, Mishra A. IL-15 immunotherapy is a viable strategy for COVID-19. Cytokine Growth Factor Rev. 2020;54:24-31. https://doi.org/10.1016/j. cytogfr.2020.06.008.

80. Mortazavi S, Kefayat A, Cai J. Low dose radiation as a treatment for COVID-19 pneumonia: a threat or real opportunity? Med Phys. 2020. https://doi.org/10.1002/mp.14367.

Publisher's Note Springer Nature remains neutral with regard to jurisdictional claims in published maps and institutional affiliations. 